\title{
Selection of modal choice nodes in urban intermodal networks
}

\author{
D. Ambrosino \& A. Sciomachen \\ DIEM - Università di Genova, Genova, Italy
}

\begin{abstract}
Urban intermodality is an increasing phenomenon and nowadays modal choice nodes, nodes within urban networks from which it is possible to reach other nodes by using different transportation modalities, play a relevant role in the analysis of passenger mobility. There is hence the need of evaluating the performance of such nodes with the aim of making more convenient multimodal paths.

In this work we focus on the problem of determining the attractive capacity of modal choice nodes in urban networks; the goal is to identify those nodes that could be attractive poles for modal exchanges and become location of new services, such as parking places, bus stops and informative panels.

Starting from relevant Origin - Destination (O-D) paths that are derived from the demand of mobility, we analyse the possible intermodal nodes by evaluating the role that such nodes play within urban networks on the basis of some structural attributes of the network itself. Inspired by the gravitational models, that are more generally used in the competitive location problems, we determine the attractive capacity of the well performing previously selected intermodal nodes; the aim is to verify whether the good behaviour of the modal choice nodes inside the transportation network is also perceived by users.

We report on some data related to the city of Genoa that for its topological and geographical structure and the wide offer of the public transportation system is well suited for intermodality.

Keywords: urban O-D paths, intermodal networks, generalized cost functions.
\end{abstract}




\section{Introduction and problem definition}

In the last decades the mobility within urban areas is changed and a more and more increasing quota of users travel along paths that involve at least two transportation modalities. We can hence say that urban passenger intermodality is now becoming a consistent phenomenon that has to be analysed (Benacchio et al. [2]).

The use of more than one transportation mode in urban network for reaching a destination node $D$ from a given origin node $O$ results in a greater value of some components of the generalized cost function and in a reduction of some others. In particular, we have higher monetary and time costs due to the modality change that is the so called transition costs, associated with a greater discomfort paid by the users; on the other hand, some travelling costs can be reduced since some economies of scales can be obtained when using different transportation modalities. A crucial role in the evaluation of the optimal trade-off between benefits and costs is plaid by the modal change nodes.

The main aim of this study is the analysis of intermodal nodes in urban transportation networks, that nowadays are not only considered as places where the change of modality occurs but also as possible locations for new attractive poles and services, such as car parks, informative panels and so on.

The location of modal change nodes must be such that it has to balance the need of reducing the intermodality costs, and hence has to be large enough for making users able to provide them all the services they need, and at the same time has to be easily reachable within the main $O-D$ paths.

In this paper we identify the possible intermodal nodes by analysing their communication capabilities with the other nodes of the network by considering both the private and the public transportation modalities; in particular, we evaluate the role that such nodes play within urban networks on the basis of their connectivity and reachability values.

The selection of the path, either intermodal or monomodal, is performed directly by the users on the basis of the foreseen travelling cost, that is given by time, monetary cost and includes some subjective elements that are not easy to be evaluated, such as social economic factors, propulsion at walking and discomfort. The shortest $O-D$ path consists hence of a path and a modal combination from $O$ to $D$ such that users have the minimum generalized cost associated with it. The final choice will be hence done on the basis of a generalized cost function with respect to both alternatives that is mono or multimodal path. Some research efforts have been already reported in the literature, mainly focusing on the shortest multimodal path problem (see, e.g., Lozano and Storchi [6], Modesti and Sciomachen [7], Ziliaskopoulos and Wardell [10]).

In the next section we present our intermodal network model and report on some data related to the central area of the city of Genoa, Italy. In Section 3 we evaluate the intermodal nodes with respect to our chosen structural attributes. In Section 4 we compute the attractivity value for all nodes that well perform from a communication point of view. 


\section{The multimodal transportation model of the city of Genoa}

Let us first present the referring urban transportation network model representing all the possible transportation modalities and the interconnections among them.

In this paper we consider the following transportation modalities: a) private, i.e. users in the private network are car drivers; b) public, i.e. users in the public network go from one location to another by bus, subway, etc.; c) pedestrian, i.e. users move into the pedestrian network by walking. Note, however, that the pedestrian modality is here considered only as a communication link between the first two modalities, as it will be explained later in more details.

As in Modesti and Sciomachen [7] we model an urban transportation network by a digraph $G=(V, E)$ that can be actually considered as the union of three subgraphs, representing, respectively, the transportation modalities listed above. More formally, $G=(V, E)$ is such that $G=G_{D} \cup G_{P} \cup G_{W}$, where $G_{D}=\left(V_{D}, E_{D}\right)$ models the private network and $G_{P}=\left(V_{P}, E_{P}\right)$ is the public network; $V_{D}$ and $V_{P}$ are, respectively, the nodes reachable by car and by the mass transit transport. $G_{W}=(P, T)$ represents the pedestrian network, where $P$ is the set of available parking places (both on the street and on buildings), and $T$ is the set of transition arcs that represent the possibility of commuting between the private and the public modalities. More formally, we consider any arc $t \in T$ as consisting of two commutation phases, namely the search for an available parking space and the walking path from the car park to either the destination node or a bus stop; consequently, we associate with each transition arc $t \in T$ a weight expressing the sum of the time required for looking for a car park and that required to go by walking, if necessary, from the car park to the bus stop together with a monetary component representing the parking tariff.

Note that arc set $T$, that takes into account also the discomfort of users, plays a crucial role in the present analysis since it represents, together with the monetary cost, the negative factor in the choice of users between the alternative of continuing the path on the same modality, that is to reach node $D$ by car, or taking the mass transit network. Therefore, the capacity and the efficacy of such arcs to be attractive enough to move people towards the intermodal network is relevant in determining the goodness of the adopted urban transportation policies for promoting the use of the local public transport.

Starting from the definition of $G$ and the available $O-D$ matrices referring to a rush hours time period, i.e. from 6.30 to 9.30 a.m., related to the city of Genoa, Italy, we have derived the corresponding network model that is reported in Figure 1; the model represents the central area of the city and is related to a linear extension of about 5 kilometres. The size of the network is the following: $|V|=56,|P|=53, E_{D}=144, E_{W}=194, E_{P}=997$. Note that the public transportation network consists in turn of a multimodal network since it is split into bus, subway, train, elevator and cableway connections. 


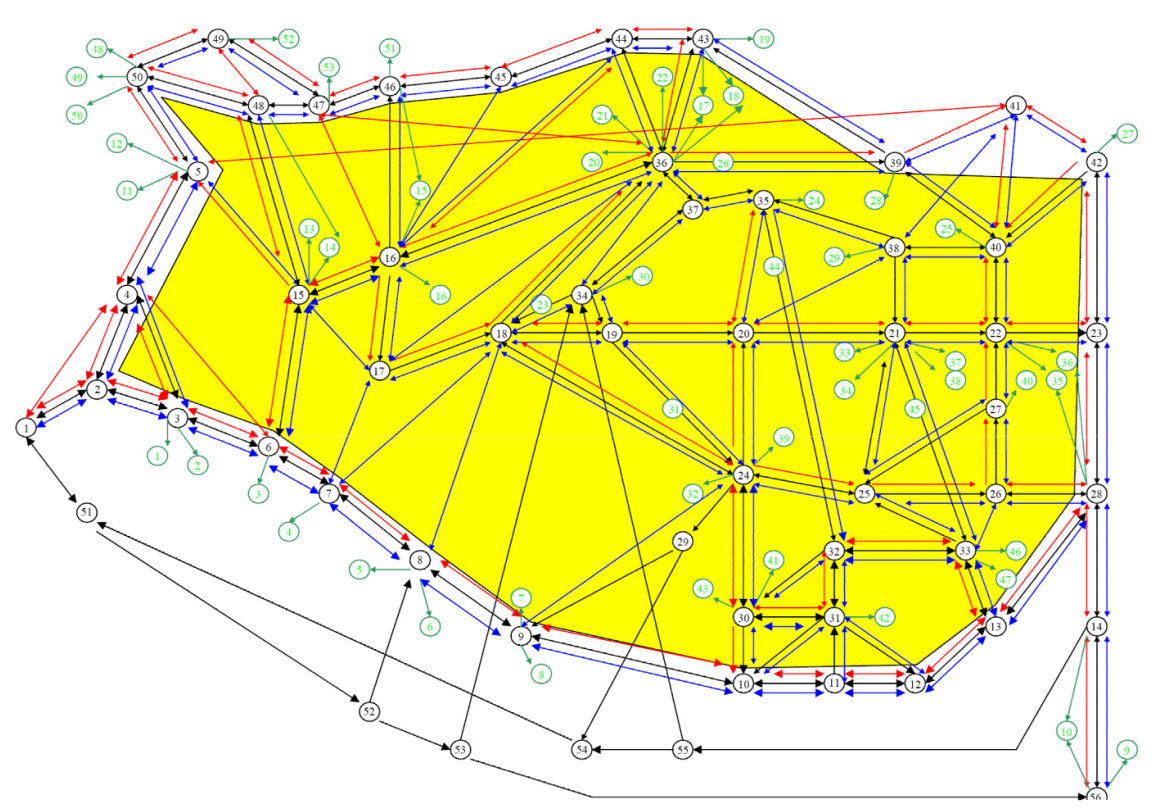

Figure 1: The intermodal transportation network of the central area of Genoa.

\section{Structural evaluation of the intermodal nodes}

Before starting our analysis let us assume that users, i.e. drivers, leave the origin node by car and have to evaluate on the way whether there is an intermodal node where they can leave the car and continue the desired path either by walking or on the public transportation network.

Since the main traffic flows in Genoa go from west to east and vice versa let us consider as origins node 1, located at the west side, and nodes 36, 42 and 56 from east (see Figure 1); we consider as destinations also the core of the city, that is nodes 18, 20, 22 and 24. In particular, let us focus our analysis on the 16 paths listed in Table 1 and see what are, if any, the intermodal nodes for such paths where it could be more convenient to change modality and continue the path on the public network with respect to a monomodal path from $O$ to $D$.

The first step of our analysis is hence to determine those nodes that are frequently visited with respect to these main $O-D$ paths on the basis of the frequency of transit through them. Note that the volume of traffic has been computed empirically by considering both the effective and the potential one, focusing the attention on the intermodal nodes that is those nodes in $G$ such that at least one entering and one outgoing arc belong to both $E_{D}$ and $E_{P}$. 
Table 1: $\quad$ Selected $O-D$ paths.

\begin{tabular}{|c|c|c|c|c|c|c|c|c|c|c|c|c|c|c|c|c|}
\hline & $\mathbf{1}$ & $\mathbf{2}$ & $\mathbf{3}$ & $\mathbf{4}$ & $\mathbf{5}$ & $\mathbf{6}$ & $\mathbf{7}$ & $\mathbf{8}$ & $\mathbf{9}$ & $\mathbf{1 0}$ & $\mathbf{1 1}$ & $\mathbf{1 2}$ & $\mathbf{1 3}$ & $\mathbf{1 4}$ & $\mathbf{1 5}$ & $\mathbf{1 6}$ \\
\hline $\mathbf{O}$ & 1 & 1 & 1 & 1 & 1 & 1 & 1 & 56 & 56 & 56 & 56 & 56 & 42 & 42 & 42 & 42 \\
\hline $\mathbf{D}$ & 36 & 24 & 40 & 22 & 32 & 20 & 18 & 36 & 24 & 32 & 20 & 18 & 24 & 32 & 20 & 18 \\
\hline
\end{tabular}

In the considered set $V$, nodes having the higher flow are those corresponding to the entering points of the network (i.e. 1, 2 and 6), and also nodes 3 and 5 that represent sorting points towards different directions. Nodes with low flow are those not easily reachable either by the public modality $(29,34-35,38,51-55)$ or by the private one (41) and those located for instance on the hill and hence visited only by few paths $(44,45)$; therefore, such nodes will be not anymore considered in our analysis.

Definition 1. We say that a node $i \in V$ is a primary intermodal node if a) $i \in V_{D}$ $\left.\cap V_{P}, b\right) i$ is connected in $G_{P}$ to at least $60 \%$ of the nodes belonging to $V$, and $c$ ) $i$ is reachable by all nodes in $V$ on $G_{D}$ along a path whose cost is at least $20 \%$ less than the average shortest path cost. $\diamond$

Definition 2. We say that a node $i \in V$ is a secondary intermodal node if a) $i \in$ $\left.V_{D} \cap V_{P}, b\right)$ either condition b) or c) of Definition 1 holds $c$ ) either condition b) or c) of Definition 1 is satisfied if it is relaxed of about $20 \%$. $\diamond$

In other words, a primary intermodal node is a node that well performs in terms of connection with the other nodes of the network by using the public modality and it is easily reachable from all other nodes by using the private modality. Secondary intermodal nodes have good performances on one of the two networks too and have a satisfactory behaviour also on the other one.

Thus our idea is to find those nodes easily reachable by car from $O$ that allow to continue the path on the public network without increasing the foreseen cost for the same required $O-D$ path on the monomodal network, that is when travelling all the path from $O$ to $D$ by car.

We then start in computing the connectivity of node $i, \forall i \in G_{D} \cap G_{P}$. That is, denoting by $r_{i}$ the number of nodes reachable by $i$ along a path that is travelled only on the public transportation network and by $n$ the number of considered nodes (i.e. in our case $|\mathrm{V}|=56$ ) we have that the connectivity value for $i$ is given by (1):

$$
c_{i}=\frac{r_{i}}{n-1}
$$

It is worth mentioning that in the computation of the connectivity value on $G_{P}$ we had to consider that the public transportation network in Genoa is a multimodal network and therefore there are paths travelled by different types of means. We assumed that no more than two means for each path from node $i$ were allowable; more precisely, we say that node $j$ is connected to node $i$ if and only if there exists a path from $i$ to $j$ in $G_{P}$ in which at most one change of mass transit mean may occur. 
For determining (1) any of the algorithms for the computation of the transitive closure on digraphs, such as the Warshall algorithm, can be used (see e.g. Hillier and Lieberman [5]).

The connectivity value of the nodes belonging to the network in Figure 1 are given in Table 2, where column $X_{i}$ reports the ratio between value $c_{i}$ of the corresponding node and the average connectivity value among all nodes; that is $X_{i}$ is given by (2) and shows how a given node performs in comparison with the other ones.

$$
\mathrm{X}_{i}=\frac{c_{i}}{\sum_{i=1, n} c_{i} / n}
$$

Table 2: $\quad$ Evaluation of the intermodal nodes of the network of Figure 1.

\begin{tabular}{|c|c|c|c|c|c|c|}
\hline $\mathbf{i}$ & $\mathbf{r}_{\mathbf{i}}$ & $\mathbf{c}_{\mathbf{i}}$ & $\mathbf{X}_{\mathbf{i}}$ & $\mathbf{d}_{\mathbf{i}}$ & $\Delta_{\mathbf{i}}$ & $\mathbf{P} / \mathbf{S}$ \\
\hline & 29 & 0,53 & 1,67 & 9,71 & 1,54 & \\
\hline 2 & 30 & 0,55 & 1,73 & 8,87 & 1,40 & \\
\hline 3 & 29 & 0,53 & 1,67 & 8,64 & 1,37 & \\
\hline 4 & 19 & 0,35 & 1,10 & 8,50 & 1,35 & \\
\hline 5 & 30 & 0,55 & 1,73 & 8,33 & 1,32 & \\
\hline 6 & 29 & 0,53 & 1,67 & 6,47 & 1,02 & \\
\hline 7 & 14 & 0,25 & 0,81 & 6,45 & 1,02 & \\
\hline 8 & 13 & 0,24 & 0,75 & 6,34 & 1,00 & \\
\hline 9 & 13 & 0,24 & 0,75 & 6,26 & 0,99 & \\
\hline 10 & 13 & 0,24 & 0,75 & 5,42 & 0,86 & \\
\hline 11 & 9 & 0,16 & 0,52 & 5,23 & 0,83 & \\
\hline 12 & 9 & 0,16 & 0,52 & 5,30 & 0,84 & \\
\hline 13 & 22 & 0,40 & 1,27 & 5,44 & 0,86 & \\
\hline 14 & 20 & 0,36 & 1,15 & 5,81 & 0,92 & \\
\hline 15 & 32 & 0,58 & 1,85 & 5,17 & 0,82 & \\
\hline 16 & 33 & 0,60 & 1,90 & 4,51 & 0,71 & $\mathrm{P}$ \\
\hline 17 & 20 & 0,36 & 1,15 & 4,89 & 0,77 & \\
\hline 18 & 38 & 0,69 & 2,19 & 4,62 & 0,73 & $\mathrm{P}$ \\
\hline 19 & 34 & 0,62 & 1,96 & 4,90 & 0,78 & $\mathrm{P}$ \\
\hline 20 & 35 & 0,64 & 2,02 & 6,72 & 1,06 & \\
\hline 21 & 34 & 0,62 & 1,96 & 5,36 & 0,85 & $\mathrm{~S}$ \\
\hline 22 & 34 & 0,62 & 1,96 & 6,19 & 0,98 & $\mathrm{~S}$ \\
\hline
\end{tabular}

\begin{tabular}{|c|l|c|c|c|c|r|}
\hline $\mathbf{i}$ & $\mathbf{r}_{\mathbf{i}}$ & $\mathbf{c}_{\mathbf{i}}$ & $\mathbf{X}_{\mathbf{i}}$ & $\mathbf{d}_{\mathbf{i}}$ & $\Delta_{\mathbf{i}}$ & $\mathbf{P} / \mathbf{S}$ \\
\hline 23 & 36 & 0,65 & 2,08 & 5,77 & 0,91 & $\mathrm{~S}$ \\
\hline 24 & 25 & 0,45 & 1,44 & 4,56 & 0,72 & $\mathbf{S}$ \\
\hline 25 & 7 & 0,13 & 0,40 & 4,85 & 0,77 & \\
\hline 26 & 6 & 0,11 & 0,35 & 5,16 & 0,82 & \\
\hline 27 & 4 & 0,07 & 0,23 & 5,27 & 0,83 & \\
\hline 28 & 21 & 0,38 & 1,21 & 5,39 & 0,85 & \\
\hline 30 & 17 & 0,31 & 0,98 & 4,86 & 0,77 & \\
\hline 31 & 13 & 0,24 & 0,75 & 5,09 & 0,81 & \\
\hline 32 & 13 & 0,24 & 0,75 & 5,07 & 0,80 & \\
\hline 33 & 13 & 0,24 & 0,75 & 5,47 & 0,87 & \\
\hline 36 & 25 & 0,45 & 1,44 & 4,74 & 0,75 & $\mathrm{~S}$ \\
\hline 37 & 20 & 0,02 & 0,06 & 5,70 & 0,90 & \\
\hline 39 & 1 & 0,02 & 0,06 & 6,10 & 0,97 & \\
\hline 40 & 24 & 0,44 & 1,39 & 5,55 & 0,88 & \\
\hline 42 & 33 & 0,60 & 1,90 & 5,88 & 0,93 & $\mathrm{~S}$ \\
\hline 43 & 17 & 0,31 & 0,98 & 7,00 & 1,11 & \\
\hline 47 & 17 & 0,31 & 0,98 & 8,02 & 1,23 & \\
\hline 48 & 7 & 0,13 & 0,40 & 6,01 & 0,95 & \\
\hline 49 & 19 & 0,35 & 1,10 & 13,11 & 2,07 & \\
\hline 50 & 29 & 0,53 & 1,67 & 8,37 & 1,32 & \\
\hline 56 & 20 & 0,36 & 1,15 & 6,19 & 0,98 & \\
\hline
\end{tabular}

Looking at Table 2 it can be easily noted that among the nodes of the intermodal transportation network of Genoa the most connected one is node 18 
that is "the heart of the city", following by the adjacent node 23 that is one of the main commercial street.

Another parameter that we consider for the classification of the intermodal nodes is their reachability, that is related to the driving distance from all nodes of the network to node $i, \forall i \in V$. For this purpose we compute the average cost from all nodes to node $i, \forall i \in G_{D}$, as given in (3), where $d_{j i} *$ is the optimal value of the solution of the shortest path problem between nodes $j$ and $i$ travelled along the private network. In this phase any algorithm proposed in the literature for solving the shortest path problem on digraphs can be used (see e. g. Gallo and Pallottino [4]).

$$
d_{i}=\frac{\sum_{i=1, n} d_{j i}{ }}{n}
$$

In the computation of the shortest path we use the usual cost functions that can be found in the transportation literature (see for instance Pallottino and Sciomachen [8], Sheffy [9]).

Values $d_{i}$ for the selected node $i$ are reported in Table 2 together with value $\Delta_{\mathrm{i}}$ that gives the performance of node $i$ in terms of reachability among the others, that is $\Delta_{\mathrm{i}}$ is given by (5):

$$
\Delta_{i}=\frac{d_{i}}{\sum_{i=1, n} d_{i} / n}
$$

Table 2 reports also the resulting primary (P) and secondary (S) intermodal nodes.

\section{Attractivity of the well performing intermodal nodes}

On the basis of the approach first presented in Ambrosino and Sciomachen [1], we then evaluated the attractive values of the resulting primary and secondary intermodal nodes. Our idea is to verify whether the nodes that well perform in term of infrastructural connections are also attractive from the users' point of view. In particular, we ask that a node is attractive enough to be such that a user is willing to leave at that location the private modality in favour of an intermodal path.

One of the basic steps when using a gravitational model is to choose those attributes that are able to reflect adequately the attractivity measure of the nodes under investigation (Drezner [3]). Therefore, the first step is to associate with each node $i \in V$ its attractivity value $a_{i}$ expressed by (5), that here we consider as depending on both the connectivity value $c_{i}$ given in (1) and the weighted sum of the considered attributed (in our case $k=6$ ) that are here below described.

$$
\mathrm{a}_{\mathrm{i}}=\frac{c_{i}}{\sum_{\mathrm{k}=1}^{6} f_{i}\left(\mathrm{x}_{\mathrm{k}}\right)}
$$


Since we are involved with urban multimodal transportation networks, we consider relevant factor the presence in the neighbourhood of node $i \in V$ of car parks (both free and payment parking places), the pedestrian modality and the public one, that is the possibility of easily reaching node $i$ on $G_{P}$. The reader can note that the first part of this evaluation hence involves arc sets $P$ and $T$. For this purpose, for each selected intermodal nodes, either primary or secondary ones, we considered the average distance from parking places, in terms of driving time from $G_{D}$ and the successive connection to $G_{P}$, that includes the waiting time for a public mean. We derived consequently the corresponding generalized cost that is computed by assuming the time conversion factor of 15 Euro / hour. For instance, the parking time for node 16 is 3.77 that will be converted in 0.94 Euro; analogously for the same node the walking time is 7.06 and the corresponding monetary cost is 1.77 . The resulting values for all nodes are given in Table 3 . Note that for each node we have the same monetary cost both for the parking tariff that is 1, 5 Euro / hour and the bus ticket, that is 1 Euro, since all of them belong to a relatively closely central area; therefore, the related attributes $x_{3}$ and $x_{4}$ will be not explicitly reported in Table 3 .

Table 3: Generalized cost of the transition phase at the corresponding intermodal nodes.

\begin{tabular}{|l|c|c|c|c|c|c|}
\hline $\mathbf{i}$ & Parking time $\mathbf{x}_{\mathbf{1}}$ & Walking time $\mathbf{x}_{\mathbf{2}}$ & $\mathbf{f}\left(\mathbf{x}_{\mathbf{1}}, \mathbf{x}_{\mathbf{2}}, \mathbf{x}_{\mathbf{3}}, \mathbf{x}_{\mathbf{4}}\right)$ & $\mathbf{f}\left(\mathbf{x}_{\mathbf{1}}-\mathbf{x}_{\mathbf{4}}, \mathbf{x}_{\mathbf{5}}\right)$ & $\mathbf{f}\left(\mathbf{x}_{\mathbf{1}}-\mathbf{x}_{\mathbf{4}}, \mathbf{x}_{\mathbf{6}}\right)$ \\
\hline 16 & 3.77 & 7.06 & $2.44+2.77=5,21$ & 5,68 & 6,10 \\
\hline 18 & 1 & 5.38 & $1,75+2.34=4,09$ & 3,77 & 4,76 \\
\hline 19 & 1.20 & 7.78 & $1.80+2.94=4.74$ & 4,89 & 5,71 \\
\hline 21 & 1.76 & 6.34 & $1.94+2.58=4,52$ & 4,74 & 5,31 \\
\hline 22 & 2.30 & 7.08 & $2.08+2.77=5,57$ & 5,14 & 5,74 \\
\hline 23 & 2.50 & 5.80 & $2.63+2.27=4,9$ & 4,72 & 5,54 \\
\hline 24 & 3.45 & 5.08 & $2.36+2.27=4,63$ & 5,06 & 5,27 \\
\hline 36 & 2.94 & 7.88 & $2.24+2.97=5,21$ & 5,58 & 6,20 \\
\hline 42 & 3.00 & 10.06 & $2.25+3.52=5,77$ & 6,15 & 7,03 \\
\hline
\end{tabular}

Successively, we analyzed some parameters that are more closely related to the attractive capacity of a node and count for the discomfort component of the users. In particular, we consider the presence of informative panels that could guaranteed to drivers the effective availability of a parking space. In fact, if there is no any parking space available in the neighbourhood of node $i$ we assume an average increase of the parking time, that is of component $x_{1}$, of about 1,5 time. Another negative attribute that we would like to consider is related to the weather condition, that in case of raining also worst the previous result as far as 
component $x_{2}$ is concerned, of about 1,5 time. The geralized cost function in both cases is also reported in Table 3 in the last two columns. The impact of the chosen impedance factors on the attractivity value of the intermodal nodes is shown in Table 4. In particular, Table 4 gives the attractivity value (5) for all primary and secondary intermodal nodes with and without considering the impedance factors.

Table 4: Attractivity values of the nodes.

\begin{tabular}{|c|c|c|c|c|}
\hline $\mathbf{i}$ & $\mathbf{a}_{1}$ & $\mathbf{a}_{\mathbf{i}}$ with $\mathbf{x}_{\mathbf{5}}$ & $\mathbf{a}_{\mathbf{i}}$ with $\mathbf{x}_{\mathbf{6}}$ & $\mathbf{1 / \mathbf { z } _ { \mathbf { i } }}$ \\
\hline 16 & 0.12 & 0.11 & 0.10 & 0.19 \\
\hline 18 & 0.17 & 0.18 & 0.14 & 0.25 \\
\hline 19 & 0.13 & 0.13 & 0.11 & 0.21 \\
\hline 21 & 0.14 & 0.13 & 0.12 & 0.22 \\
\hline 22 & 0.11 & 0.12 & 0.11 & 0.18 \\
\hline 23 & 0.13 & 0.14 & 0.12 & 0.20 \\
\hline 24 & 0.10 & 0.09 & 0.09 & 0.22 \\
\hline 36 & 0.09 & 0.08 & 0.07 & 0.19 \\
\hline 42 & 0.10 & 0.10 & 0.09 & 0.17 \\
\hline
\end{tabular}

We can observe that without any penalty factor the most attractive node is node 18 that is a primary intermodal node, followed by nodes 21,19 and 23 . Note that node 18 always performs better than the other nodes since in all columns its value is the minimum one and also in an uncomfortable condition it still remains an attractive location. However, it is worth mentioning that the discomfort attribute strongly affect the attractivity value of the nodes that rank last in the first case.

The last column of Table 4 shows how the ranking of the nodes changes whenever the connectivity attribute is not considered in the attractivity evaluation; in particular, we can note that node 24 would move in this case at the second best position. This last consideration is of course just a qualitative evaluation of the performance of the intermodal nodes without considering their very relevant position within the network.

As a last consideration we have to say that by computing the shortest path for all O-D pairs given in Table 1, only in one case, that is for path 1-40, the intermodality results to be the best choice for users, reducing the total cost of about $12 \%$. However, the chosen intermodal node is really node 18 that is our most favorite intermodal nodes. This last result confirms the validity of the proposed approach in the evaluation of the intermodal nodes with the hope that in a next future among the considered paths more than in one case the intermodal network would allow reducing the travel cost. 


\section{References}

[1] Ambrosino, D., Sciomachen, A., A gravitational approach for locating new services in urban areas. In Advanced $O R$ and AI Methods in transportation, Proc. Euro Working Group on Transportation, Poznan, Publishing House of Poznan University of Technology, 194-199, 2005.

[2] Benacchio, M., Musso, E., Sciomachen, A., Intermodal transport in urban passenger mobility. In Urban transport and the environment for the 21st century, IV, C. Borrego, L. Sucharov (eds.), Southampton-Boston: Wessex Institute of Technology Press, Computational Mechanics Publications, 247-257, 1998.

[3] Drezner, T., Locating a Single New Facility Among Existing Unequally Attractive Facilities. J. Regional Science, 34, 237 - 252, 1994.

[4] Gallo G. Gallo, Pallottino S. Shortest path algorithms, Annals of Operations Research, V. 13, p. 3, 1988.

[5] Hillier, F.S., Lieberman, G.J, Introduction to Operations Research, McGraw-Hill, NY, 2005.

[6] Lozano, A., Storchi, G., Shortest viable path in multimodal networks. Transportation Research Part A, 35, 225-241, 2001.

[7] Modesti, P., Sciomachen, A., A utility measure for finding multiobjective shortest paths in urban multimodal transportation networks. European Journal of Operational Research, 111/3, 495-508, 1998.

[8] Pallottino, S., Sciomachen, A., Scienze delle decisioni per $i$ trasporti. FrancoAngeli, Milano, 1999.

[9] Sheffy, Y., Urban transportation networks. Prentice - Hall, Englewood Cliffs, NY, 1985.

[10] Ziliaskopoulos A., Wardell A. An intermodal optimum path algorithm for multimodal networks with dynamic arc travel times and switching delays. European Journal of Operational Research, 125, 486-502, 2000. 